\title{
Posicionamento do Departamento de Ergometria, Exercício, Cardiologia Nuclear e Reabilitação Cardiovascular (DERC/SBC) sobre a Atuação Médica em suas Áreas Durante a Pandemia por COVID-19
}

\author{
Position Statement of the Brazilian Society of Cardiology Department of Exercise Testing, Sports Exercise, \\ Nuclear Cardiology, and Cardiovascular Rehabilitation (DERC/SBC) on Activities Within its Scope of Practice \\ During the COVID-19 Pandemic
}

Realização: Departamento de Ergometria, Exercício, Cardiologia Nuclear e Reabilitação Cardiovascular (DERC) da Sociedade Brasileira de cardiologia (SBC)a (SBA), Associação Médica Brasileira (AMB)

Conselho de Normatizações e Diretrizes (2020-2021): Brivaldo Markman Filho, Antonio Carlos Sobral Sousa, Aurora Felice Castro Issa, Bruno Ramos Nascimento, Harry Correa Filho, Marcelo Luiz Campos Vieira

Coordenador de Normatizações e Diretrizes (2020-2021): Brivaldo Markman Filho

Autores do Posicionamento: Gabriel Blacher Grossman, ${ }^{1,2}$ Carlos Alberto Cyrillo Sellera, ${ }^{3,4}$ Carlos Alberto Cordeiro Hossri, ${ }^{5,6}$ Lara Terra F. Carreira, ${ }^{7,8}$ Antônio Carlos Avanza Jr.,,${ }^{9,10}$ Pedro Ferreira de Albuquerque, ${ }^{11,12}$ Mauricio Milani, ${ }^{13 @}$ Luiz Eduardo Mastrocola, ${ }^{5}{ }^{\circledR}$ Luiz Eduardo Fonteles Ritt, ${ }^{14,15}$ Odilon Gariglio Alvarenga de Freitas, ${ }^{16}$ Tales de Carvalho, ${ }^{17,18}$ William Azem Chalela, ${ }^{19,20}$ Nabil Ghorayeb, ${ }^{5,6}$ Romeu Sergio Meneghelo, ${ }^{6,21}$ R Mauricio Batista Nunes, ${ }^{22}$ Salvador Manoel Serra ${ }^{23}$

Hospital Moinhos de Vento, ${ }^{1}$ Porto Alegre, RS - Brasil

Clínica Cardionuclear, ${ }^{2}$ Porto Alegre, RS - Brasil

Santa Casa de Misericórdia de Santos, ${ }^{3}$ Santos, SP - Brasil

Faculdade de Medicina da Universidade Metropolitana de Santos, ${ }^{4}$ Santos, SP - Brasil

Hospital do Coração (Hcor), ${ }^{5}$ São Paulo, SP - Brasil

Instituto Dante Pazzanese de Cardiologia, ${ }^{6}$ São Paulo, SP - Brasil

Hospital Nossa Senhora do Pilar, ${ }^{7}$ Curitiba, $P R-$ Brasil

Cardiologia Nuclear Curitiba, ${ }^{8}$ Curitiba, $P R-$ Brasil

Universidade Vila Velha, ${ }^{9}$ Vitória, ES - Brasil

Clínica Centrocor, ${ }^{10}$ Vitória, ES - Brasil

Universidade Federal de Alagoas, ${ }^{11}$ Maceió, AL - Brasil

Hospital Memorial Arthur Ramos, ${ }^{12}$ Maceió, AL - Brasil

Clínica Fitcordis, ${ }^{13}$ Brasília, DF - Brasil

Escola Bahiana de Medicina e Saúde Pública, ${ }^{14}$ Salvador, BA - Brasil

Hospital Cardiopulmonar, ${ }^{15}$ Salvador, BA - Brasil

Minascor Centro Médico, ${ }^{16}$ Belo Horizonte, MG - Brasil

Clínica de Prevenção e Reabilitação Cardiosport, ${ }^{17}$ Florianópolis, SC - Brasil

Universidade do Estado de Santa Catarina, ${ }^{18}$ Florianópolis, SC - Brasil

Instituto do Coração (Incor) do Hospital das Clínicas da Faculdade de Medicina da Universidade de São Paulo (HCFM-USP), ${ }^{19}$ São Paulo, SP - Brasil Sociedade Beneficente de Senhoras Hospital Sírio-Libanês, ${ }^{20}$ São Paulo, SP - Brasil

Hospital Israelita Albert Einstein, ${ }^{21}$ São Paulo, SP - Brasil

Hospital Português, ${ }^{22}$ Salvador, BA - Brasil

Centro de Cardiologia do Exercício do Instituto Estadual de Cardiologia Aloysio de Castro (CCEx/IECAC), ${ }^{23}$ Rio de Janeiro, RJ - Brasil

Esta diretriz deverá ser citada como:

Grossman GB, Sellera CAC, Hossri CAC, Carreira LTF, Avanza Jr. AC, Albuquerque PF, et al. Posicionamento do Departamento de Ergometria, Exercício, Cardiologia Nuclear e Reabilitação Cardiovascular (DERC/SBC) sobre a atuação médica em suas áreas durante a pandemia por COVID-19. Arq Bras Cardiol. 2020; 115(2):284-291

Nota: Estes Posicionamentos se prestam a informar e não a substituir o julgamento clínico do médico que, em última análise, deve determinar o tratamento apropriado para seus pacientes.

Correspondência: Sociedade Brasileira de Cardiologia - Av. Marechal Câmara, 360/330 - Centro - Rio de Janeiro - CEP: $20020-907$.

E-mail: diretrizes@cardiol.br

DOI: https://doi.org/10.36660/abc.20200797 


\begin{tabular}{|c|c|c|c|c|c|c|c|}
\hline \multicolumn{8}{|c|}{$\begin{array}{l}\text { Declaração de potencial conflito de interesses dos autores/colaboradores do Posicionamento do Departamento de Ergometria, Exercício, Cardiologia Nuclear } \\
\text { e Reabilitação Cardiovascular (DERC/SBC) sobre a Atuação Médica em suas Áreas Durante a Pandemia por COVID-19 } \\
\text { Se nos últimos } 3 \text { anos o autor/colaborador do Posicionamento: }\end{array}$} \\
\hline $\begin{array}{l}\text { Nomes Integrantes } \\
\text { do Posicionamento }\end{array}$ & $\begin{array}{c}\text { Participou de } \\
\text { estudos clínicos e/ } \\
\text { ou experimentais } \\
\text { subvencionados } \\
\text { pela indústria } \\
\text { farmacêutica ou } \\
\text { de equipamentos } \\
\text { relacionados ao } \\
\text { posicionamento em } \\
\text { questão }\end{array}$ & $\begin{array}{l}\text { Foi palestrante } \\
\text { em eventos } \\
\text { ou atividades } \\
\text { patrocinadas } \\
\text { pela indústria } \\
\text { relacionados ao } \\
\text { posicionamento em } \\
\text { questão }\end{array}$ & $\begin{array}{l}\text { Foi (é) membro } \\
\text { do conselho } \\
\text { consultivo ou } \\
\text { diretivo da indústria } \\
\text { farmacêutica ou de } \\
\text { n equipamentos }\end{array}$ & $\begin{array}{l}\text { Participou } \\
\text { de comitês } \\
\text { normativos de } \\
\text { estudos científicos } \\
\text { patrocinados pela } \\
\text { indústria }\end{array}$ & $\begin{array}{l}\text { Recebeu auxílio } \\
\text { pessoal ou } \\
\text { institucional da } \\
\text { indústria }\end{array}$ & $\begin{array}{l}\text { Elaborou textos } \\
\text { científicos em } \\
\text { periódicos } \\
\text { patrocinados pela } \\
\text { indústria }\end{array}$ & $\begin{array}{l}\text { Tem ações da } \\
\text { indústria }\end{array}$ \\
\hline $\begin{array}{l}\text { Antônio Carlos } \\
\text { Avanza Jr. }\end{array}$ & Não & Não & Não & Não & Não & Não & Não \\
\hline $\begin{array}{l}\text { Carlos Alberto } \\
\text { Cordeiro Hossri }\end{array}$ & Não & Não & Não & Não & Não & Não & Não \\
\hline $\begin{array}{l}\text { Carlos Alberto } \\
\text { Cyrillo Sellera }\end{array}$ & Não & Não & Não & Não & Não & Não & Não \\
\hline $\begin{array}{l}\text { Gabriel Blacher } \\
\text { Grossman }\end{array}$ & Não & Não & Não & Não & Não & Não & Não \\
\hline $\begin{array}{l}\text { Lara Terra F. } \\
\text { Carreira }\end{array}$ & Não & Não & Não & Não & Não & Não & Não \\
\hline $\begin{array}{l}\text { Luiz Eduardo } \\
\text { Fonteles Ritt }\end{array}$ & Não & Não & Não & Não & Não & Não & Não \\
\hline $\begin{array}{l}\text { Luiz Eduardo } \\
\text { Mastrocola }\end{array}$ & Não & Não & Não & Não & Não & Não & Não \\
\hline $\begin{array}{l}\text { Mauricio Batista } \\
\text { Nunes }\end{array}$ & Não & Não & Não & Não & Não & Não & Não \\
\hline Mauricio Milani & Não & Não & Não & Não & Não & Não & Não \\
\hline Nabil Ghorayeb & Não & Não & Não & Não & Não & Não & Não \\
\hline $\begin{array}{l}\text { Odilon Gariglio } \\
\text { Alvarenga de } \\
\text { Freitas }\end{array}$ & Não & Não & Não & Não & Não & Não & Não \\
\hline $\begin{array}{l}\text { Pedro Ferreira de } \\
\text { Albuquerque }\end{array}$ & Não & Não & Não & Não & Não & Não & Não \\
\hline $\begin{array}{l}\text { Romeu Sergio } \\
\text { Meneghelo }\end{array}$ & Não & Não & Não & Não & Não & Não & Não \\
\hline $\begin{array}{l}\text { Salvador Manoel } \\
\text { Serra }\end{array}$ & Não & Não & Não & Não & Não & Não & Não \\
\hline Tales de Carvalho & Não & Não & Não & Não & Não & Não & Não \\
\hline $\begin{array}{l}\text { William Azem } \\
\text { Chalela }\end{array}$ & Não & Não & Não & Não & Não & Não & Não \\
\hline
\end{tabular}




\section{Posicionamento}

\section{Sumário}

1. Regras Gerais 286

2. Teste Ergométrico e Teste Cardiopulmonar de Exercício ....... 286

3. Reabilitação Cardiopulmonar e Metabólica. 287

4. Cardiologia Nuclear ............................................................. 288 4.1. Adaptação da Prática da Cardiologia Nuclear Durante a Pandemia .... 288 4.1.1. Considerações Gerais no Agendamento do Exame................... 288 4.1.2. Considerações no Momento da Chegada do Paciente ao Serviço .... 288

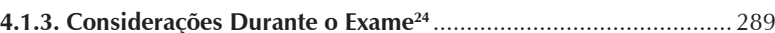

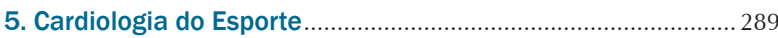

5.1. Atividade Físico-Esportiva na Pandemia por COVID-19 ................... 289

5.2. Atividade Física em Residência ................................................. 289

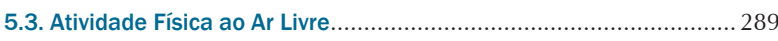

5.4. Atividades Físicas em Academias ................................................. 290

5.5. Fui Acometido por COVID-19 - Quando Posso Voltar à Atividade

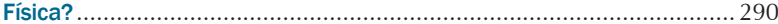

5.6. Avaliação de Atletas que Foram Acometidos por Infecção por CoVID-19....

Referências 290

\section{Regras Gerais}

- O Departamento de Ergometria, Exercício, Cardiologia Nuclear e Reabilitação Cardiovascular (DERC) acompanha atentamente a pandemia por COVID-19 e suas consequências, encontrando-se alinhado com a Associação Médica Brasileira (AMB), com as posições publicadas pelos departamentos especializados e sociedades filiadas.

- Reconhece que a contenção da pandemia é de estratégia fundamental.

- Este documento reúne de forma atualizada as recomendações para minimizar os riscos dos pacientes e a exposição dos executores durante esse período pandêmico.

- Dada a dinâmica da pandemia, qualquer dessas recomendações poderá ser atualizada caso surjam novos fatos e evidências científicas.

- Todas as medidas preventivas orientadas pelo Ministério da Saúde (MS) e Organização Mundial de Saúde (OMS) deverão ser adotadas de forma sistemática com cuidados de alta qualidade para pacientes com doenças cardiovasculares, por serem considerados de elevados riscos.

- Todo e qualquer procedimento deve respeitar as normas preconizadas de higienização, uso de equipamento de proteção individual (EPI) e restrição de contatos.

- Eventual suspensão, continuação ou interrupção de atividades inerentes às áreas do SBC/DERC devem observar as determinações das autoridades sanitárias locais ou as normas internas das instituições de saúde.
- A remuneração dos exames de teste ergométrico (TE), teste cardiopulmonar de exercício (TCPE), cardiologia nuclear e reabilitação cardiopulmonar, no momento, não poderá sofrer redução em decorrência das medidas tomadas pela pandemia, salvo orientação em contrário da Câmara Técnica Permanente da Classificação Brasileira Hierarquizada de Procedimentos Médicos da AMB.

\section{Teste Ergométrico e Teste Cardiopulmonar de Exercício}

- Avaliação cuidadosa de queixas e sintomas respiratórios e de outros quadros infecciosos agudos desde o contato telefônico para marcação e na confirmação ou não do exame, de modo a evitar a saída desnecessária dos pacientes em locais onde haja distanciamento social. Evitar a vinda de acompanhantes, exceto nos exames de menores de 18 anos e incapazes. ${ }^{1,2}$

- O paciente que já tiver apresentado COVID-19 e encontrarse recuperado, assintomático e estável clinicamente deve postergar a realização do TE e TCPE por, no mínimo, 30 dias após o quadro. Mesmo após a COVID-19, o paciente deverá seguir todas as recomendações e procedimentos descritos neste documento.

- Considerando os riscos potenciais de geração de contaminantes durante TE e TCPE, recomendamos reduzir o número de exames o máximo possível - idealmente, um por hora por ergômetro.

- Após a confirmação do agendamento, orientar os pacientes para virem com as roupas e calçados adequados, pois não haverá possibilidade de utilização de vestiários nas clínicas e hospitais.

- Na chegada do paciente, realizar a reavaliação dos sintomas (preenchimento de questionário epidemiológico específico ou entrevista). Verificar a temperatura corporal além de fornecer máscara cirúrgica logo na entrada do serviço de saúde. As recepcionistas e secretárias deverão usar máscara facial e luvas durante todo o tempo, bem como manter distância segura dos pacientes atendidos. ${ }^{3,4}$

- De modo habitual, aplicar termo de consentimento livre e esclarecido, que é obrigatório.

No entanto, sugere-se adição de considerações complementares em função da pandemia vigente: não é possível precisar acuradamente quais os riscos quantitativos de adquirir o coronavírus em um TE e TCPE, mas:

- As medidas preventivas possíveis serão tomadas para minimizar a contaminação.

- Há, provavelmente, maior risco de contrair infecção durante TE e TCPE em relação a um exame fora da pandemia.

- O médico executante deve contextualizar de forma adequada as indicações dos exames e, em casos suspeitos de COVID-19 ou de outra síndrome respiratória aguda (história de febre, tosse, coriza, astenia, taquicardia, cianose, alterações da ausculta pulmonar), comunicar ao médico assistente e suspender a realização do exame. 
- A solicitação de TE e TCPE requer, durante a consulta, um exame físico completo do paciente antes da indicação dos mesmos. Devido a essa condição, não é possível a solicitação desses exames através de consultas por telemedicina. ${ }^{5}$

- As salas de realização de exame devem ser amplas e ventiladas. Preferir ambientes com ventilação natural, evitando os sistemas de climatização de ambiente comuns (ventilador e ar-condicionado) devido ao risco potencial destes em dispersar contaminantes no ambiente. ${ }^{6}$

- Sabe-se que o TE e TCPE são exames de risco teórico de contaminação para o médico executante e equipe executora. Sugere-se que os médicos executores e auxiliares (técnico de enfermagem, paramédicos, enfermeiras) usem, a cada exame, máscara com filtração mínima equivalente à PFF2/N95, óculos de proteção e luvas de procedimentos. Manter o maior tempo possível um distanciamento físico do paciente superior a 2 metros. Sugere-se observar as recomendações institucionais e das secretarias municipais e estaduais da saúde.

- O paciente deve usar máscara com filtração mínima equivalente à PFF1, como as máscaras cirúrgicas, desde sua entrada na área de exames. O paciente deverá higienizar as mãos por meio de lavagem prévia com água e sabão e álcool em gel $70 \%$ antes de contato com qualquer superfície e equipamentos existentes na sala de exame.

- Em exames realizados em clínicas e hospitais, antes do início do exame, confirmar realização de higienização e limpeza dos aparelhos e superfícies potencialmente contaminantes. Seguir os protocolos institucionais e que contemplem as recomendações das autoridades sanitárias referentes a esses procedimentos. ${ }^{6}$

No caso de realização de exames em consultórios ou clínicas que não tenham os protocolos instituídos, recomenda-se:

- Fazer limpeza comum do cabo do aparelho de ECG do TE/TCPE com um tecido embebido em álcool a 70\%.

- Realizar limpeza e desinfecção para qualquer patógeno transmissível na barra de apoio do ergômetro, tapete da esteira, selim do cicloergômetro, manguito do esfigmomanômetro, estetoscópio e outras superfícies de contato utilizando um ou mais dos produtos recomendados: $:^{1,7}$

- Com base no hipoclorito de sódio (solução de hipoclorito de sódio ativo a 0,5\%).

- Com base em amônia quaternária (QUAT), tomando o cuidado de que a concentração total para o uso deve ser menor que $0,8 \%$.

- Com base no peróxido de hidrogênio acelerado a, no máximo, 0,5\%.

- À base de álcool 70\% ou álcool e amoníaco quaternário (QUAT).

- Preferencialmente, utilizar materiais descartáveis para realização de TE e TCPE, em especial, quanto aos eletrodos de monitoramento. Descartar todos os materiais de maneira adequada e em local apropriado.
- No caso do TCPE, o médico executante deve confirmar a capacidade efetiva de esterilização de todo o sistema de condução e análise dos gases expirados, além de seguir os protocolos institucionais que contemplem as recomendações das autoridades sanitárias.

- O médico executante deverá se atualizar, verificar e adequar o material de emergência e reanimação cardiorrespiratória, de modo a adequar às novas recomendações para atendimentos de intercorrências e complicações durante a COVID-19.8

- Os serviços de TE e TCPE deverão atualizar seus protocolos de transferência de pacientes em caso de intercorrências e emergências, de acordo com a disponibilidade e orientações dos convênios, cooperativas de saúde e órgãos públicos de socorro. ${ }^{10}$

- Os profissionais (médico executante e auxiliares) com suspeita e diagnóstico de COVID-19 devem ser afastados das atividades, seguirem tratamento e isolamento recomendados.

- Manter os critérios de escolha de ergômetros e protocolos de esforço, os clássicos critérios diagnósticos e prognósticos do TE e do TCPE e as condições pré e pós-analíticas (probabilidades) tradicionalmente utilizadas. Sugerimos descrever no laudo o comportamento do intervalo QT no esforço e no quarto minuto da recuperação. ${ }^{11,12}$

- No momento atual, é razoável considerar o adiamento da realização de TE e TCPE nos casos em que, provavelmente, não impactará diretamente nos cuidados ou nos resultados clínicos nos próximos meses. ${ }^{3}$

\section{Reabilitação Cardiopulmonar e Metabólica}

A COVID-19 tem causado profundo impacto nos serviços de saúde, inclusive nos serviços de reabilitação cardiopulmonar e metabólica (RCPM), fundamentais no manejo clínico dos pacientes com doenças cardiovasculares, pneumopatias e doenças metabólicas, que proporcionam significativas reduções nas taxas de internações hospitalares e mortalidade geral. ${ }^{13-17}$

No entanto, no momento, o isolamento social tem sido a pedra angular no controle da COVID-19, especialmente dos pacientes de maior risco para internação hospitalar, complicações respiratórias e mortalidade, que são justamente aqueles com indicação para os programas de RCPM. ${ }^{18,19}$ Portanto, em sintonia com as recomendações das autoridades sanitárias mundiais e nacionais, devido ao risco de contágio, os serviços de RCPM com atividades presenciais foram interrompidos.

No contexto da COVID-19, sendo a RCPM imperativa, por exemplo, em processos de recuperação da capacidade funcional de pacientes com insuficiência cardíaca ${ }^{17,20}$ ou após eventos e intervenções cardiovasculares, quando o tempo de início dos exercícios após a alta hospitalar pode influenciar na recuperação funcional, controle da doença e redução da mortalidade, consideramos que devem ser priorizados os programas de RCPM a distância, baseados em domicílio, com o apoio do uso de tecnologia digital, que têm sido adotados com bons resultados iniciais por muitos serviços nacionais e internacionais. ${ }^{17,21}$ 


\section{Posicionamento}

Os exercícios domiciliares devem seguir as recomendações habituais da RCPM convencional, com prescrições individualizadas, sempre que possível baseadas em avaliações prévias. ${ }^{17,21}$ Por segurança, orienta-se que, durante os exercícios físicos, seja considerada a escala de percepção de esforço, com recomendação de intensidade leve e/ou moderada. No momento atual, sugerimos que sejam evitados exercícios de alta intensidade, muito desgastantes, com percepção de esforço muito elevado (muito forte).

Ressalta-se que, diante da heterogeneidade nacional da curva epidemiológica dos casos de COVID-19, peculiaridades regionais, incidências de novos casos e necessidades de internações, além de aspectos relacionados com infraestrutura e taxa de ocupação dos serviços de saúde públicos e privados, diferentes recomendações podem ser pertinentes nas localidades, sempre de acordo com as orientações das organizações e autoridades sanitárias. ${ }^{22}$

Assim que sinais de controle da pandemia forem evidentes, havendo maior flexibilização do isolamento social pelas autoridades sanitárias, os serviços convencionais de RCPM com atividades presenciais poderão retomar suas atividades de forma gradual e com a rigorosa observância aos cuidados pertinentes de proteção dos pacientes e profissionais de saúde. Na ocasião do reinício paulatino das atividades, recomendamos:

- Pacientes, profissionais da equipe assistencial e acompanhantes com sintomas gripais ou contato com casos confirmados/suspeitos nos últimos 14 dias devem se manter afastados pelo prazo recomendado pelas organizações e autoridades sanitárias. ${ }^{23}$

- Na triagem dos pacientes que chegam ao serviço, é recomendada a medida de temperatura na região frontal por infravermelho (sem contato cutâneo).

- Uso de máscara facial, álcool em gel e lavagem das mãos com água e sabão são recomendados como obrigatório pelos pacientes e demais frequentadores do ambiente de exercícios, sendo que os profissionais da equipe assistencial devem seguir as determinações dos órgãos de saúde, sindicatos e conselhos profissionais em relação ao uso de EPI.

- Disponibilização de álcool a 70\% em spray e papéis descartáveis para higienização dos equipamentos de exercícios, antes e após o uso individual, devendo ser evitado o uso compartilhado de equipamentos em circuitos de treinamento (aparelhos de musculação, pesos livres, espaldares e outros).

- Promoção de maior circulação de ar nas salas de exercícios, mantendo, sempre que possível, portas e janelas abertas.

- Redução da quantidade de pacientes atendidos simultaneamente, possibilitando maior distanciamento entre eles.

- Adoção de horários predefinidos de atendimentos, com duração rigorosamente controlada, com intervalos entre as sessões, a fim de evitar a sobreposição de grupos e permitir higienização do ambiente e equipamentos.

Observação: visando à proteção jurídica dos serviços, recomenda-se a solicitação de carta de encaminhamento ao programa de reabilitação do médico assistente, bem como a exigência da assinatura de termo de consentimento após esclarecimento pelos pacientes.

\section{Cardiologia Nuclear}

A orientação para os serviços de cardiologia nuclear durante a pandemia é de que se realizem apenas os estudos urgentes e em pacientes sintomáticos, quando o resultado do exame tiver o potencial de alterar o manejo evolutivo imediato ou que possa impactar o prognóstico do paciente a curto prazo. Também se faz urgente a avaliação de pacientes internados e de pronto atendimento, objetivando direcionar a conduta, reduzir o tempo de internação e expandir a capacidade hospitalar, ${ }^{3,24}$

\subsection{Adaptação da Prática da Cardiologia Nuclear Durante a Pandemia}

\subsubsection{Considerações Gerais no Agendamento do Exame ${ }^{24,25}$}

- Aumentar o intervalo entre os exames para evitar aglomerações.

- No ato do agendamento, perguntar se o paciente apresenta sinais ou sintomas sugestivos de possível infecção por COVID-19 (febre, tosse, dispneia, fadiga incomum, mialgia, diarreia, anosmia, hiposmia, disgeusia ou ageusia). Em caso afirmativo, de preferência, adiar o agendamento.

- Perguntar se o paciente foi exposto a algum caso confirmado ou suspeito nas 2 semanas anteriores. Em caso afirmativo, de preferência, postergar a marcação do exame.

- Torna-se importante entrar em contato com os pacientes no dia anterior ao exame para garantir que não estejam apresentando sinais ou sintomas suspeitos. Em caso afirmativo, reagendar o exame se possível.

- Os pacientes devem ser instruídos a comparecer ao exame sozinhos. Caso seja necessário o acompanhante, vir com apenas uma pessoa, idealmente sem fatores de risco de relevância como diabetes, cardiopatias não estáveis, arritmias, idosos > 65 anos de idade, entre outros.

- Solicitar para que os pacientes e acompanhantes venham usando EPIs (máscaras faciais como requisito mínimo) ou considerar fornecê-las para serem usadas durante todo o tempo que estiverem no serviço de medicina nuclear.

\subsubsection{Considerações no Momento da Chegada do Paciente ao Serviço ${ }^{24-26}$}

- Na chegada ao laboratório nuclear, deve-se questionar novamente o paciente quanto à presença de sintomas e exposição à COVID-19 (por meio do preenchimento de questionário epidemiológico específico e/ou entrevista).

- Dado o risco de transmissão por portadores assintomáticos, a equipe de atendimento ao paciente na sala de espera e demais funcionários não médicos no laboratório devem usar máscara o tempo todo.

- Solicitar para que os pacientes e acompanhantes permaneçam com as máscaras faciais enquanto estiverem no serviço de medicina nuclear. 
- As instalações devem garantir que as salas de espera tenham fácil acesso à lavagem das mãos e/ou álcool em gel.

- Manter pelo menos 2 metros de distância entre as pessoas, evitando aglomerações nas salas de espera e instalações do serviço. Orientar que sigam as regras de espaçamento, etiqueta respiratória e lavagem frequente das mãos e/ou álcool gel.

- Evitar a interação entre pacientes internados e ambulatoriais, bem como a de pacientes oncológicos e imunocomprometidos nos casos de serviços que realizem mais de uma modalidade de exame.

\subsubsection{Considerações Durante o Exame ${ }^{24-26}$}

\section{A) Com relação à equipe de trabalho e ambiente}

- Aplicar os princípios gerais de uso de EPIs da saúde durante todo o exame.

- Minimizar o número de funcionários em contato com o paciente.

- Minimizar o tempo de contato paciente/equipe.

- Reforçar a higienização frequente das mãos.

- Se o paciente apresentar sintomas suspeitos, a equipe em contato com ele deverá usar EPI completo (máscara, proteção ocular, avental e luvas) e fornecer uma máscara ao paciente.

- Em pacientes com COVID-19 ativo confirmado, qualquer teste deve ser feito apenas se absolutamente necessário. Consultar as políticas locais de controle de infecção e considerar agendamento como último estudo do dia e em equipamento separado, se possível. Após o exame, uma limpeza terminal completa deve ser realizada na sala e equipamentos.

- Gantry, maca, esteira, equipamentos de pressão arterial, estetoscópio e bombas de infusão devem ser limpos após cada exame por pessoal com EPI apropriado.

- É mandatória a realização de limpeza regular das superfícies de contato incluindo maçanetas, superfícies de mesa, computadores, teclados, telefones e equipamentos de ditado por funcionário usando EPI apropriado.

\section{B) Seleção do protocolo de exame 24}

- Selecionar o protocolo de menor duração.

- Considerar protocolos de imagem de um dia.

\section{C) Seleção do protocolo de estresse ${ }^{24}$}

- Como o vírus SARS-CoV-2 é transmitido por gotículas, os procedimentos que envolvem a produção de gotículas ou aerossóis são considerados de maior risco. Sendo assim, o estresse farmacológico é preferido ao TE.

- Se o TE for considerado necessário, a equipe deve usar os EPIs (preferencialmente, máscara N95/PFF2) e manter distância do paciente quando não estiver prestando a assistência direta ou injetando o radiofármaco - seguir as orientações deste documento quanto à realização do TE.
- Manguitos automáticos para medida da pressão arterial devem ser considerados.

\section{D) Interpretação do exame ${ }^{24-26}$}

- Evitar vários médicos e/ou estagiários no mesmo local, se possível.

- Nos exames em que se realiza a tomografia computadorizada para a correção de atenuação, as imagens devem ser interpretadas no contexto de possíveis achados pulmonares de COVID-19.

\section{Cardiologia do Esporte}

\subsection{Atividade Físico-Esportiva na Pandemia por COVID-19}

A atividade física regular é essencial para promoção de saúde e correção dos fatores de risco para doenças cardiovasculares, e o sedentarismo piora a evolução e aumenta a mortalidade das doenças cronicodegenerativas. Tanto para o isolamento compulsório como no caso da haver liberação sanitária para as pessoas se deslocarem livremente, listamos orientações para prática de atividade física em domicílio, para academias, em ambientes ao ar livre e esportes em geral. ${ }^{27}$

\subsection{Atividade Física em Residência}

De forma geral, as seguintes orientações devem ser seguidas: ${ }^{27}$

- Praticar exercícios em locais ventilados, mantendo, sempre que possível, janelas e portas abertas.

- Se mais de uma pessoa for se exercitar no mesmo ambiente, manter distância mínima de 2 metros, ou seja, uma pessoa a cada $4 \mathrm{~m}^{2}$.

- De preferência, a prática de atividade física deve ser feita individualmente e, por segurança, executar os exercícios a que esteja habituado.

- Realizar higienização completa com água e sabão ou álcool em gel (70\%) das mãos e equipamentos durante a atividade física.

- Usar e trocar toalhas individuais descartáveis ou de tecido.

- Controlar o esforço dispendido nos treinos, com as recomendações previamente estabelecidas pelo seu médico, evitando excessos físicos.

- Suspender os exercícios caso surja algum sintoma: cansaço, dor no peito ou nas costas, tonturas, palpitações, dores musculares, febre, náuseas, vômitos, diarreia ou outros sintomas de estado gripal.

- Sedentários ou destreinados há muito tempo das atividades físicas só devem realizar atividades físicas leves.

\subsection{Atividade Física ao Ar Livre}

Obrigatório seguir as orientações da autoridade de saúde local quanto às restrições da prática ao ar livre. ${ }^{27}$ Onde as medidas de restrição forem reduzidas, recomenda-se a forma individualizada e isolada, com os devidos cuidados antes referidos. Devemos ter em mente que, no curto prazo, não 
há tratamento específico para o vírus, e algumas medidas de cuidado devem ser mantidas.

Atualmente, não existem muitos padrões validados de recomendações específicas para a prática de atividade ao ar livre em uma pandemia. Apenas um estudo belgo-holandês sugeriu que a distância de 2 metros é ineficaz para evitar a propagação do vírus, e sugere:

1. Distância de 4 a 5 metros, a ser obedecida entre as pessoas que andam uma atrás da outra.

2. Distância de 10 metros, ao correr ou andar de bicicleta lentamente.

3. Distância de 20 metros, ao andar de bicicleta rapidamente.

Devemos ressaltar que as medidas adotadas, assim como as condutas sugeridas, sofrem constantes mudanças de acordo com o cenário da pandemia. ${ }^{28-30}$

\subsection{Atividades Físicas em Academias}

- Disponibilizar álcool em gel a 70\% e máscaras faciais para uso dos alunos e colaboradores em todas as áreas da academia (recepção, musculação, peso livre, salas de coletivas, piscina, vestiários, etc.).

- Sugere-se controle ativo de temperatura na entrada da academia.

- Limpeza geral e desinfecção dos ambientes por 30 minutos, uma a duas vezes/dia.

- Kits de limpeza em pontos estratégicos das áreas de musculação e peso livre, contendo toalhas de papel para descarte imediato após uso e produto específico de higienização dos equipamentos de treino: colchonetes, halteres e máquinas.

- Limitar a quantidade de alunos na academia e o espaço em que cada aluno deve se exercitar, nas áreas de pesos livres e nas salas de atividades coletivas onde a ocupação simultânea será a cada 4 m² (p. ex., áreas de treino, vestiário).

- Deixar o espaçamento de um equipamento sem uso para o outro.

- Liberar a saída de água no bebedouro somente para uso de garrafas próprias.
- Academias dos condomínios/residenciais: sendo liberadas pelas autoridades sanitárias, recomenda-se reservar horário exclusivo para os moradores da mesma unidade habitacional. Após o uso, é obrigatório realizar medidas de limpeza adequadas. ${ }^{29}$

\subsection{Fui Acometido por COVID-19 - Quando Posso Voltar à Atividade Física?}

Qualquer que seja a atividade física regular escolhida, só deve ser reiniciada após negativação da PCR e liberação clínica. As atividades físico-esportivas de qualquer intensidade necessitam da avaliação médica de pré-participação, objetivando diagnóstico de possíveis sequelas. ${ }^{30-34}$

\subsection{Avaliação de Atletas que Foram Acometidos por Infecção por COVID-19}

- Atletas com infecção assintomática e presença de anticorpo confirmada.

- Atletas com histórico de doença leve (sem hospitalização) relacionado com COVID-19, confirmado ou suspeito.

- Atletas com histórico de doença moderada a grave (com hospitalização) relacionado com COVID-19, confirmado ou suspeito.

- Atletas com histórico de infecção por COVID-19 (independentemente da gravidade) com lesão miocárdica confirmada por um ou mais dos seguintes exames: alterações no ECG hospitalar, elevação de troponina ultrassensível ou peptídio natriurético, arritmia ou função cardíaca comprometida.

Obrigatório realizar a avaliação pré-participação (APP) com ECG e demais exames complementares de acordo com a avaliação inicial. Sempre que possível, comparar com exames anteriores, com foco para rastrear achados pós-infecciosos persistentes ou novos.

No retorno ao treinamento, no caso de atletas que tenham apresentado alterações em exames cardiológicos quando acometidos por COVID-19, as imagens cardíacas em série serão necessárias, sendo tal retorno gradual. Além disso, devido ao acometimento cardíaco, deve ser acompanhado por um especialista.

\section{Referências}

1. Sociedade Brasileira de Cardiologia. (SBC) Recomendações ao Cardiologista para minimizar os riscos de exposição durante a pandemia de COVID-19.2020, 23 março. [Acesso em 24 março 2020] Disponível em: https://www.portal. cardiol.br/post/comunicado-da-diretoria-de-qa-da-sbc-minimizando-aexposi\%C3\%A7\%C3\%A3o-do-cardiologista-\%C3\%A0-covid-19.

2. Associação Médica Brasileira (AMB) Diretrizes AMB: COVID-19. 09 de abril de 2020. [Acesso em 09 de abril 2020] Disponível em: https://amb.org.br/ wp-content/uploads/2020/04/DIRETRIZES-AMB-COVID-19-atualizadoem-09.04.2020.pdf.

3. Gluckman TJ; American College Of Cardiology. General Guidance on Deferring Non-Urgent CV Testing and Procedures During the COVID-19

Pandemic. ACC Clinical Guidance and Practice. Mar 24, 2020. [Acesso em 24 março 2020] Disponível em: https://www.acc.org/latest-in-cardiology/ articles/2020/03/24/09/42/general-guidance-ondeferring-non-urgent-cvtesting-and-procedures-during-the-covid-19-pandemic

4. Centers for Disease Control and Prevention (CDC) Infographic: Sequence for Putting on Personal Protective Equipment (PPE). [Acesso em 03 março 2020] Disponível em: https://www.cdc.gov/hai/pdfs/ppe/PPE-Sequence.pdf

5. European Society of Cardiology (ESC) ESC Guidance for the Diagnosis and Management of CV Disease during the COVID-19 Pandemic. [Acesso em 28 maio 2020]. Disponível em: https://www.escardio.org/Education/COVID19-and-Cardiology/ESC-COVID-19-Guidance 
6. Colégio Brasileiro de Radiologia e Diagnóstico por Imagem. (CRB) Recomendações gerais de prevenção de infecção pelo COVID-19 para clínicas e serviços hospitalares de diagnóstico por imagem. [Acesso em 14 março 2020. Disponível em: https://cbr.org.br/wp-content/ uploads/2020/03/CBR_Recomenda\%C3\%A7\%C3\%B5es-gerais-depreven\%C3\%A7\%C3\%A30-de-infec\%C3\%A7\%C3\%A3o-pelo-COVID19-para-cl\%C3\%ADnicas-e-servi\%C3\%A7os-hospitalares-de-diagn\%C3\%B3stico-por-imagem.pdf

7. Conselho Federal de Medicina. Posição sobre a pandemia de COVID-19: contexto, análise de medidas e recomendações. 17 de março de 2020. [Acesso em 18 março 2020]. Disponível em: http://portal.cfm.org.br/images/ PDF/covid-19cfm.pdf

8. Associação Brasileira de Medicina de Emergência (ABRAMEDE) Recomendações para Intubação Orotraqueal em pacientes portadores de COVID-19. Associação Brasileira de Medicina de Emergência (ABRAMEDE), Associação de Medicina Intensiva Brasileira (AMIB), Sociedade Brasileira de Cardiologia (SBC), Conselho Latino Americano de Emergências Cardiovasculares e Ressuscitação (CLARE). [Acesso em 15 abril 2020] Disponível em: https://abramede.com.br/recomendacoes-para-iot-empacientes-portadores-de-covid-19/.

9. Edelson DP, Sasson C, Chan PS, Atkins DL, Aziz K, Becker LB, et al. Interim Guidance for Basic and Advanced Life Support in Adults, Children, and Neonates With Suspected or Confirmed COVID-19: From the Emergency Cardiovascular Care Committee and Get With the Guidelines - Resuscitation Adult and Pediatric Task Forces of the American Heart Association in Collaboration with the American Academy of Pediatrics, American Association for Respiratory Care, American College of Emergency Physicians, The Society of Critical Care Anesthesiologists, and American Society of Anesthesiologists: Supporting Organizations: American Association of Critical Care Nurses and National EMS Physicians. Circulation. 2020 Apr 09 [Acesso em 10 abril 2020]. Disponível em: CIRCULATIONAHA.120.047463

10. Shah PB, Welt FGP, Mahmud E, Phillips A, Kleiman NS, Young MN, et al. Triage Considerations for Patients Referred for Structural Heart Disease Intervention During the Coronavirus Disease 2019 (COVID-19) Pandemic: An ACC/SCAI Consensus Statement. JACC Cardiovasc Interv. abril de 2020 [acesso em 20 abril 2020]. Disponível em: https://doi.org/10.1016//j.jcin-2020.04.001

11. Meneghelo RS; Araújo CGS; Stein R; Mastrocolla LE; Albuquerque PF; Serra SM et al. III Diretrizes da Sociedade Brasileira de Cardiologia sobre Teste Ergométrico. Arq Bras Cardiol. 2010; 95(5 supl 1):1-26. [Acesso em 12 março 2020] Disponível em: https://doi.org/10.1590/S0066782X2010000800001

12. Serra, SM, Lima RSL. Teste ergométrico, teste cardiopulmonar de exercício, cardiologia nuclear, reabilitação cardiopulmonar e metabólica, cardiologia do esporte e do exercício: o livro do DERC. Rio de Janeiro: Elsevier; 2020.

13. Anderson L, Oldridge N, Thompson DR, Zwisler AD, Rees K, Martin N, et al. Exercise-Based Cardiac Rehabilitation for Coronary Heart Disease: Cochrane Systematic Review and Meta-Analysis. J Am Coll Cardiol. 2016; 67(1):1-12.

14. Taylor RS, Sagar VA, Davies EJ, Briscoe S, Coats AJ, Dalal H, et al. Exercisebased rehabilitation for heart failure. Cochrane Database Syst Rev. 2014(4):CD003331.

15. Herdy AH, Lopez-Jimenez F, Terzic CP, Milani M, Stein R, Carvalho T, et al. Consenso Sul-Americano de Prevenção e Reabilitação Cardiovascular. Arq Bras Cardiol. 2014; 103(2 Suppl 1):1-31.

16. Carvalho T, Milani M, Ferraz AS, Silveira AD, Herdy AH, Hossri CAC, et al. Diretriz Brasileira de Reabilitação Cardiovascular - 2020. Arq Bras Cardiol. 2020; 114(5):943-98

17. Adawi KT, Supervia M, Lopez-limenez F, Contractor A, Grace SL. Cardiac rehabilitation availability and density around the globe. EClinicalMedicine. 2019; 13:31-45. 13: 31-45.

18. Yeo T J, Wang YTL, Low T T. (2020). Have a heart during the COVID-19 crisis: Making the case for cardiac rehabilitation in the face of an ongoing pandemic. Eur J Prev Cardiol. 2020.

19. Wong, JEL, Leo, YS, Tan, CC. COVID-19 in Singapore-current Experience. Critical global issues that require attention and action. JAMA. Epub ahead of print 20 February 2020. DOI: 10.1001/jama.2020.2467.
20. Haykowsky M, Scott J, Esch B, Schopflocher D, Myers J, Paterson I, et al. A meta-analysis of the effects of exercise training on left ventricular remodeling following myocardial infarction: start early and go longer for greatest exercise benefits on remodeling. Trials. 2011;12:92.

21. Zhang YM, Lu Y, Tang Y, Yang D, Wu HF, Bian ZP, et al. The effects of different initiation time of exercise training on left ventricular remodeling and cardiopulmonary rehabilitation in patients with left ventricular dysfunction after myocardial infarction. Disabil Rehabil. 2016; 38(3):268-76.

22. Anderson L, Sharp GA, Norton RJ, Dalal H, Dean SG, Jolly K, et al. Homebased versus centre-based cardiac rehabilitation. Cochrane Database Syst Rev. 2017; 6:CD007130.

23. Driggin E, Madhavan MV, Bikdeli B, Chuich T, LaracyJ, Bondi-Zoccai G, etal Cardiovascular Considerations for Patients, Health Care Workers, and Health Systems During the Coronavirus Disease 2019 (COVID-19) Pandemic. J Am Coll Cardiol. 2020; 75(18):2352-71.

24. Skali H, Murthy VL, Mouaz H. Al-Mallah MH,Mouaz H, Baterman TM, Beanlands R, et al. Guidance and Best Practices for Nuclear Cardiology Laboratories during the Coronavirus Disease 2019 (COVID-19) Pandemic: An Information Statement from ASNC and SNMMI. J Nucl Cardiol. 2020 May 15:1-8.

25. Paez D, Gnanasegaran G, Fanti S, BOMANJI m, Hacker M, Satherkge HS, et al. COVID-19 pandemic: guidance for nuclear medicine departments, Eur J Nucl Med Mol Imaging. 2020 Apr 15:1-5 doi: https://doi.org/10.1007/ s00259-020-04825-8

26. Loke KSH, Tham WY, Bharadwaj P, Keng F, Huang Z, Bin Idu M, et al Adapting to a novel disruptive threat; Nuclear Cardiology Service in the Time of the Coronavirus (COVID-19) Outbreak 2020 (SARS REBOOT). J Nucl Cardiol. 2020 Apr 19; 1-5 Online ahead of print.

27. American College of Sports Medicine. Staying Physically Active During COVID-19 pandemic. 2020 Mar 16. [Acesso em 22 maio 2020 ] Disponível em; https://www.acsm.org/read-research/newsroom/news-releases/newsdetail/2020/03/16/staying-physically-active-during-covid-19-pandemic

28. Niiler E. Are Running or Cycling Actually Risks for Spreading Covid-19? Science. 2020; 84:14

29. Mann RH, Clift BC, Boykoff J, Beker S. Athletes as community; athletes in community: covid-19, sporting mega- events and athlete health protection. Br J Sports Med. 2020 Apr 17; bjsports-2020-102433.; 0:1-2. doi:10.1136/ bjsports-2020-102433.

30. Baggish AL, Drezner JA, Kim JH, Martinez MW, Prutkin JM. The resurgence of sport in the wake of COVID-19: cardiac considerations in competitive athletes. (Blog) Br J Sports Med. 2020 Apr 24.

31. Phelan D, Kim JH, Chung EC. A game plan for the resumption of sport and exercise after coronavirus disease 2019 (COVID-19) infection. JAMA Cardiol. JAMA Cardiol. 2020 May 13. Doi: 10.1001/jamacardiol.2020.2136. On line ahead of print.

32. Maron BJ, Udelson JE, Bonow RO, Nishimura RA, Ackerman MJ Estes NA 3rd,et al. Eligibility and disqualification recommendations for competitive athletes with cardiovascular abnormalities: task force 3: hypertrophic cardiomyopathy, arrhythmogenic right ventricular cardiomyopathy and other cardiomyopathies, and myocarditis: a scientific statement from the American Heart Association and American College of Cardiology. Circulation. 2015; 132(22):e273-80.

33. Pelliccia A, Solberg EE, Papadakis M,AdamiPE, Biffi A, Caselli S, et al, Recommendations for participation in competitive and leisure time sport in athletes with cardiomyopathies, myocarditis, and pericarditis: position statement of the Sport Cardiology Section of the European Association of Preventive Cardiology (EAPC). Eur Heart J. 2019 Jan 1; 40(1):19-33.

34. Ghorayeb N, Stein R, Daher DJ, Silveira AD, Ritt LEF, Santos DFP, et al. Atualização da Diretriz em Cardiologia do Esporte e do Exercício da Sociedade Brasileira de Cardiologia e da Sociedade Brasileira de Medicina do Esporte - 2019. Arq Bras Cardiol. 2019; 112(3):326-68. 


\section{Edição de Agosto de 2020, vol. 115 (2), págs. 284-291}

No "Posicionamento do Departamento de Ergometria, Exercício, Cardiologia Nuclear e Reabilitação Cardiovascular (DERC/SBC) sobre a Atuação Médica em suas Áreas Durante a Pandemia por COVID-19", com número de DOI: https://doi. org/10.36660/abc.20200797, publicado no periódico Arquivos Brasileiros de Cardiologia, 115(2): 284-291, na página 290, onde se lia:

"Qualquer que seja a atividade física regular escolhida, só deve ser reiniciada após negativação da PCR e liberação clínica."

O correto é:

"Para pessoas previamente diagnosticadas com Covid-19 sintomático, que permanecem assintomáticos após a recuperação, um novo teste não é recomendado dentro dos próximos 3 meses após a data do início da infecção, e a liberação para atividade física dependerá da liberação clínica." 